\title{
EP-191
}

\section{Long-term oncologic benefit of postoperative chemother- apy in resected ampulla of Vater cancer}

\author{
Sukwon CHANG', Chang Moo KANG*1 \\ 'Division of HBP Surgery, Department of Surgery, Yonsei University College of Medicine, Seoul, Korea \\ ${ }^{2}$ Pancreatobiliary Cancer Center, Yonsei Cancer Center, Severance Hospital, Seoul, Korea
}

Introduction: The oncological effects of adjuvant chemotherapy after surgery for resected ampulla of Vater cancer (AoVCa) remain controversial. This study was conducted to confirm the oncological effects of adjuvant chemotherapy after surgery in patients who underwent radical surgery with AoVCa.

Methods: For this study, data about clinical pathological characteristics, postoperative chemotherapy, and long-term survival from 306 AoVCa patients who underwent surgical pancreaticoduodenal resection from 2005 to 2019 were retrospectively and reviewed.

Results: Patients were classified into groups that received adjuvant chemotherapy $(\mathrm{n}=124,40.5 \%)$ and those who did not $(\mathrm{n}=182$, 59.5\%). There were significant differences in Cancer stage $(p<0.001)$, lymph node involvement $(p<0.001)$, PNI $(p<0.001)$, LVI $(p<$ $0.001)$, and cancer differentiation $(p=0.010)$. As a result, relatively low long-term survival $(p<0.001)$ was found in the group received adjuvant chemotherapy. As a result of multivariate analysis based on univariate analysis of patient's survival, factors affecting the patient's prognosis include cancer progression, Lymph node involvement (HR $=3.50$ [95\% CI: 1.76-6.93], $p<0.001)$, PNI (HR $=1.90$ [95\% CI: 1.07-3.40], $p=0.029$ ), LVI ( $\mathrm{HR}=1.83$ [95\% CI: 1.00-3.36], $p=0.052$ ), cancer differentiation, but the presence of adjuvant chemotherapy does not have a significantly affect the patient's long-term survival rate. The regimen of chemotherapy did not show any significant statistics related to patient survival $(p=0.629)$.

Conclusions: The oncological effect of adjuvant chemotherapy after surgery in resected AoVCa patients is not clear and will remain controversial in the future. In order to improve the therapeutic outcome of resected AoVCa, it is urgent to develop effective anticancer treatment methods for patients with risk factors. 